\title{
Red transfronteriza en turismo: \\ una exploración en Ciudad \\ Juárez, Chihuahua, México, \\ EI Paso, Texas, Estados Unidos
}

DOI: $10.22403 /$ UQROOMX/TYP08/08

\author{
Isabel Zizaldra Hernández \\ Universidad Autónoma de Ciudad Juárez \\ Departamento de Ciencias Administrativas, \\ Licenciatura en Turismo
}

\section{$|h|+\mid$}

Resumen

El presente documento es un estudio sobre las relaciones turísticas binacionales México-Estados Unidos dentro de un contexto transfronterizo, su incipiente potencialidad reticular, en una actividad socioeconómica excluida por la visión de emprendedores y el ámbito público; afectado por la falta de comunicación entre actores y stakeholders en ambos lados de la frontera. Entre los beneficios del examen de redes se encuentra la identificación de los bloques participantes, la viabilidad del desarrollo de un destino común y sus posibilidades de integración a una red en turismo regional entre Ciudad Juárez (CJ) y El Paso (ELP).

\begin{tabular}{r|} 
Palabras \\
Clave
\end{tabular} Red, turismo transfronterizo, México-Estados Unidos 


\section{Introducción}

Las relaciones fronterizas entre México y Estados Unidos no han estado exentas de tensiones. Sucesos como la independencia de Texas (I836), las cuatro guerras o la negociación para definir los actuales límites fronterizos (1964), que se extienden por casi 3200 kilómetros entre límites artificiales y naturales, y van del Golfo de México al Océano Pacífico, han influido en las relaciones internacionales entre ambos países a lo largo de la historia. Si bien en el siglo $X I X$, las relaciones fueron tensas durante el $x X$ y lo que va del XXI, aunque con ciertos roces, se han preservado dentro de una política internacional y de comercio admisibles. Como Giménez concibe:

La frontera es simplemente una línea político-administrativa que se mide en términos de longitud, pero no de anchura. La franja fronteriza, en cambio, es un territorio. Ambas nociones son, por supuesto, indisociables, pero constituyen conjuntamente el marco de lo que podríamos llamar fenómenos fronterizos [2007: 20].

Con el comienzo del siglo xxI, Ciudad Juárez (c) ya había definido su naturaleza económica al igual que las principales ciudades fronterizas del Norte. Finalmente, el programa de manufactura iniciado en el siglo pasado a finales de 1960 había logrado sus frutos. La actividad socioeconómica giraba plácidamente alrededor del concepto de twin plant, y los tres niveles de gobierno centraban cada vez más su entusiasmo por incrementar el número de plantas, garantizando el desarrollo en la franja fronteriza.

Sin embargo, no es hasta los acontecimientos terroristas del II de septiembre que se percibieron los primeros síntomas de una política exclusivista en torno a un solo sector económico. Asimismo se da la apertura de China al mundo occidental, y el hecho de que su gobierno ofrecía amplias garantías a las empresas de manufactura implicaba sobre todo una reducción en los costos de operación. Ambas situaciones repercutieron de manera significativa en las comunidades fronterizas, y el entusiasmo público se transformó por primera vez, después de décadas de crecimiento, en preocupación.

La demanda laboral bajó e igualmente empresas instaladas con cierto arraigo empezaron a ver la oportunidad de emigrar a Asia. El contexto, en definitiva, estaba cambiando, ciertamente en primera instancia por cuestiones macroeconómicas, pero en el fondo existían condiciones desventajosas para las empresas de manufactura, por ejemplo los beneficios laborales (bonos de 
transporte, cafetería, asistencia, productividad, etc.) incrementaban los costos de operación, así como la reiterada problemática de la burocracia legal, limitante para la instauración de compañías en México. De modo que la ubicación geoestratégica en la frontera México-Estados Unidos no era el único elemento que considerar.

Estos acontecimientos dejaron ver la insuficiencia del modelo económico apoyado en un solo sector marcado por la dependencia, haciéndolo sobre todo vulnerable y sensible. Por lo tanto, un modelo más híbrido en las relaciones socioeconómicas y político-económicas incluyendo a la actividad turística puede conducir a una expectativa más equilibrada de desarrollo en la frontera MéxicoEstados Unidos.

\section{Cambio económico en la frontera norte}

Como actividad, el turismo es una de las tres grandes fuentes de divisas en México, por lo tanto implica progreso económico en aquellos espacios que lo han adoptado. El alcance del turismo supone un entramado constituido por los diferentes sectores económicos; su naturaleza compleja ofrece alternativas de desarrollo utilizando la infraestructura apoyada en el patrimonio natural y cultural regional, además de que amplía las expectativas de calidad de vida al crear nuevas oportunidades de empleo y profesionalización. Aunque, refiriéndose al turismo fronterizo, el escándalo ha sido el común denominador en su clasificación porque se ha vinculado con la depravación, drogas, prostitución y negocios ilícitos; condiciones que se replican generalmente en las fronteras del mundo, con la agravante de ser consideradas violentas e inseguras, y cj no ha sido la excepción.

A principios del siglo $x x$, c cambió su base económica. Volvió sus ojos al turismo, ya que su comercio próspero había sido arruinado con la abolición de la zona libre, su agricultura se había visto seriamente afectada por la escasez de agua y su industria había sido dañada por los obstáculos que tenía el comercio interno. La construcción de centros turísticos destacados, como la plaza de toros en 1903 y 1905, reflejó esta transformación; en adelante, las diversiones predominaron en la vida de la ciudad. Así dio inicio la época del escándalo (Martínez, 1982) que ha estigmatizado a la ciudad, principalmente por los sucesos de carácter criminal. Sin embargo, en la actualidad es imperativo 
reconsiderar el potencial estratégico del turismo en las regiones fronterizas, que por la dimensión de las oportunidades es amplio,y por las posibilidades de diversificación económica es plausible. Sus implicaciones son manifiestas en la calidad medioambiental y en el desarrollo regional,ya que se apoya en la riqueza del paisaje, el pasado histórico, la cultura y el idioma, factores determinantes de viabilidad turística, como apoyo al modelo económico vigente. Porque las fronteras abiertas

...facilitan los flujos económicos y culturales, propician el pendularismo cotidiano entre las zonas fronterizas de uno y otro lado, y estimula[n] la creación de organizaciones transfronterizas, como las que existen entre regiones francesas, belgas, alemanas y el pequeño Estado de Luxemburgo en Europa [Giménez, 2007: 21].

Por estas consideraciones el turismo fronterizo amerita reevaluarse, pues ya existen signos de su importancia, como las propuestas que ha ofrecido el alcalde de El Paso (ELP) John Cook en su plan de gobierno (City of El Paso Museums and Cultural Affairs Department Strategic Agency); entre ellas el programa "Ciudades creativas", que ha generado acciones encaminadas a hacer de ELP el destino receptor y distribuidor de turismo cultural en la región. En tanto, la Secretaría de Desarrollo Comercial y Turístico considera para Juárez el impulso del turismo de negocios.Así, el análisis del turismo transfronterizo en red es deseable para identificar la participación concienzuda de actores-stakeholders en las comunidades fronterizas de C y ELP, así como las alianzas dentro de la actividad en un entorno interdependiente. De igual modo, el análisis es importante para evaluar las relaciones entre stakeholders y líderes, con objeto de integrar la información en un escenario transfronterizo.

No obstante, históricamente las comunidades de CJ y ELP han explorado la actividad turística transfronteriza, ya sea mediante la mercadotecnia de sus potenciales, ya sea Juárez al vender a El Paso,Texas por sus centros comerciales y outlets, o El Paso,Texas, al ofrecer a Juárez en sus promociones para conocer una parte de México. De manera que pensar en un entorno mixto en las relaciones socioeconómicas y sociopolíticas articulado por el turismo transfronterizo es factible, aunque se encuentra condicionado a las tensiones derivadas del crimen y el terrorismo.

El tratamiento del turismo transfronterizo puede considerarse enigmático, pero existen medidas recomendables dentro del argumento del desarrollo regional y la interdependencia compleja que constituyen paradigmas por solucionar. 


\section{Enigma del turismo transfronterizo}

Para comprender y explorar el turismo transfronterizo es imperativo conocer los conceptos de nación y soberanía, que parten de otro más remoto: la identidad de los pueblos. Cabe aclarar que la aproximación a estas nociones será desde el concepto de identidad. Respecto a ésta, Laura Bolaños (200I: 47) dice que "se forma en el transcurso de una historia compartida dentro de un territorio, con idioma, religión e idiosincrasia comunes, sin descontar con la variación a que dan las formaciones locales". Además, es menester el acercamiento al vocablo transfronterizo, referido por el prefijo "trans" como "paso al lado opuesto" o "situación en el lado opuesto" (Moliner, 1998: I280), y el sustantivo frontera, que señala el "límite o confín de un Estado" (Moliner, 1998: I 280). Ambas definiciones no logran precisar el argumento transfronterizo. De modo que el tema explora los medios requeridos para estandarizar las relaciones socioeconómicas en comunidades fronterizas que por su cercanía necesitan construir un vínculo, para estar mejor articuladas y responder a una coexistencia ineludible.

Las características de las relaciones transfronterizas económicas y sociales se encuentran, en términos generales, en investigaciones contemporáneas.Timothy y Butler (1995) y Timothy (1995) analizan la actividad transfronteriza como generadora de turismo, donde la participación de la iniciativa privada y el sector público es determinante para el progreso del comercio, fundamentalmente de las compras, juegos en pueblos fronterizos, centros de bienvenida y enclaves internacionales (World Trade Center). Los entornos contemplados son las viejas fronteras, parques, monumentos, recursos naturales localizados en límites fronterizos y fronteras subnacionales, los cuales influyen en políticas legislativas, sociales y económicas.

A su vez, Lagiewski y Revelas (2002) efectúan su exploración desde la perspectiva de relaciones colaborativas entre organizaciones del orden público y privado en ambientes internacionales tensos, por ejemplo en el sureste Adriático, donde ven, sin embargo, la posibilidad de beneficios. En este sentido, loannides, Nielsen y Billing (2006) señalan intereses comunes en un destino común. Los stakeholders tienen la capacidad de influir en el turismo, aun en condiciones no favorables, como sucede en Suecia y Finlandia. Son contrastantes los trabajos de Trevor y Sofield (2006) y Prokkola (2007) en el sureste asiático y en el Círculo Polar Ártico, al referir apoyo a proyectos de cooperación transnacional con 
perspectivas de desarrollo turístico mediante el uso de nuevas tecnologías y movilidad.

Interdependencia socioeconómica compleja

El concepto de interdependencia, de acuerdo con el Diccionario Ideológico de la Lengua Española ( 1998: I 252), significa "dependencia recíproca” o "dependencia mutua entre personas, entidades, naciones, principios". La interdependencia compleja entraña el uso de canales múltiples y brinda la posible ausencia de jerarquía; también es precedente de normas de coexistencia entre actores internacionales. Mientras, el precepto propuesto por Keohane y Nye (I 988:23) establece que las relaciones interdependientes siempre implicarán costos, dado que la interdependencia reduce la autonomía; pero es imposible determinar a priori si los beneficios de una relación serán mayores que los costos. No obstante, nada asegura que las relaciones interdependientes no alcancen a ser de beneficio mutuo.

Como observa Martínez (1994:28), la interdependencia se encuentra ligada a la evolución de la frontera mexicana-americana desde 1920 y hasta la fecha; a partir de entonces se distingue, por ejemplo, la ratificación de los acuerdos, como el tratado del agua, la entrega del Chamizal, el acuerdo comercial a la expansión industrial en la frontera, turismo y migración. Asimismo apunta que uno de los sectores económicos en la frontera es el turismo, el cual se destaca por los negocios de entretenimiento y de trismo de negocio que constituyen una de las fuentes de ingreso y empleo para la población local (Martínez, 1994: 5 I).

Desde este panorama se pueden comprender relaciones transgubernamentales, interestatales y transnacionales; escenarios proclives al entorno transfronterizo (Keohane y Nye, 1988). Empero, en este tejido el poder está influenciado por factores políticos, culturales, tecnológicos, económicos y del manejo de la información, constantes en la globalización. Por su parte, Welch y Wong (200I), al interpretar el entorno doméstico con relación a la globalización en los países, establecen la actuación burocrática a través de un cambio en los sistemas económicos, políticos y sociales necesarios en el supuesto de la interdependencia compleja.

Esta regulación promovida desde la interdependencia compleja, mediante la existencia de normas de cooperación que impliquen más satisfacer la coexistencia, tiene alcances de consenso y cooperación en las relaciones reticulares 
que surgen como propuestas político-estratégicas con efectos socioeconómicos. Dado que Juárez ha supeditado su desarrollo a su vecina estadounidense, El Paso, y con el crecimiento económico y demográfico cíclico debido a las migraciones, ha profundizado los déficit en infraestructura, equipamiento, vivienda y servicios (Méndez y Rodríguez, 2004). Por ello es menester procurar una mayor atención al impacto potencial directo del turismo a través de un destino turístico común que permita generar una dinámica diferente.

\section{Actores o stakeholders}

"La relación entre individuos y sociedad es algo único. No es análogo con otra esfera de la existencia”, dice Norbert Elías (200l: I8) quien sugiere además la necesidad de considerar a cada participante como un todo, porque la integración de sus miembros determina la estructura de las relaciones y, por tanto, su participación en conjunto. El autor declara que "es necesario pensar en términos individuales, aislar la sustancia y comenzar pensando en términos de relaciones y funciones" (Elías, 200 I: 19). Con esta concepción, se torna complicado clasificar a los stakeholders en turismo, aunque, como señala Coleman: "Pero no importa qué tan sinceras sean [las relaciones] Lo cierto en las relaciones sociales es qué tan sustentables sean los incentivos en ambas partes, para continuar con la relación intrínseca” (Coleman, 1994: 43).

Otro escenario para la comprensión de actores -stakeholders es estudiar la sociabilidad, la forma en que las personas se interconectan entre sí forjando relaciones entre identidades diferenciadas, subrayando facetas particulares de sus propias identidades y articulándolas con facetas complementarias de las identidades de otros, lo cual podría hacerse a través de un origen regional compartido, por ejemplo, al experimentar problemas comunes (Gledhill, 1999:45).

Sólo con observar los ámbitos en los que se desenvuelven los actoresstakeholders en turismo, se percibe la necesidad de reciprocidad en acciones concretas o de incentivos, porque sus acciones van desde el nivel global, subglobal, nacional, local y en el destino. La labor se extiende como intermediaria en la actividad no solamente en la administración pública, asociaciones sociales, proveedores de empresas turísticas, estructuras científicas y académicas, sino en los socios público-privado y privado-público, aparte de las sociedades civiles y organismos no gubernamentales, en la frontera. 
Durante décadas la cooperación transfronteriza se ha utilizado para la compensación de las desventajas competitivas de los mercados turísticos. El ejemplo de la nueva AlpNets proyecto demuestra la importancia de la cooperación y los miembros son los requisitos específicos para el turismo y otros sectores económicos en lo que se refiere a la creación de redes de conocimiento [Pechlaner,Abfalder y Raich 2002: 89-90].

Cada uno de ellos contribuye con diversos ordenamientos para la gestión de políticas interdestinos en los que se encuentran los servicios de movilidad y transporte, los proveedores de productos o los que suministran un servicio (viajes, hospedaje, alimentación), así como aquellos que se enfocan a la consultaría, la investigación o los medios de comunicación (European Comm Unities, 2006: I30).

\section{Redes transfronterizas}

De acuerdo con Vázquez Barquero (1999:98), la red "puede definirse como el sistema de relaciones y/o contactos que vinculan a las empresa/actores entre sí, cuyo contenido puede referirse a bienes materiales, información o tecnología". Y continúa diciendo:"Desde la perspectiva de la actividad económica se trataría de relaciones de empresas o empresarios que permiten el intercambio de bienes y servicios o de aquellas informaciones que incorporan conocimiento" (Vázquez Barquero, 1999: 98). La mirada también puede partir del motivo intrínseco de cada nodo, porque la reflexión de redes transfronterizas parte de la complejidad de delinear escenarios colaborativos. Si bien Watts (1999), en SmallWorlds, se basa en la concepción del gráfico caveman, esta simplicidad en su configuración e interconexión tiene implícito el obstáculo de la apertura colaborativa. Porque "el proceso de sociabilidad, empero, está también y siempre imbuido ya de relaciones de poder" (Gledhill, 1999:45). Por su parte, Heijden (1996, cit. en Werthner y Klein, 1999: I8), apoyado en la intermediación turística denominada alternative network structures, ofrece una aproximación donde señala tres tipos de redes en turismo que dan muestras del contenido estratégico al ejemplificar el proceso durante la transacción de servicios.

Con base en la aproximación de diagramas estratégicos se han bosquejado cuatro caracterizaciones de contextos posibles en el desarrollo de redes transfronterizas en turismo, con la finalidad de identificar no sólo la viabilidad, sino el potencial reticular. Con este criterio, la exploración proyecta seis escenarios: I) 
dispersión en la frontera, 2) configuración de las primeras redes, 3) tejido por intereses comunes, 4) noción reticular local e influencia fronteriza, 5) noción reticular local y regional, y 6) estado reticular ideal en la articulación transfronteriza.

Este proceso evolutivo en las redes transfronterizas va pasando en diferentes fases. Claro que esta evolución no ocurre de manera lineal, pero en la aproximación de manera holística se identifican tres escenarios dinámicos: I) dinámica de dispersión a brote o integración de redes, 2) dinámica de tejido por intereses a noción reticular local, y 3 ) dinámica noción reticular local y regional al estado ideal.

La región transfronteriza de CJ-ELP (véase figura I) debe cuestionarse la viabilidad de las expectativas del turismo como una actividad centrada en un territorio compartido, que permita el desarrollo de las comunidades en ambos lados de la frontera; para ello, es menester preguntar ¿cuáles son las barreras que enfrentan las redes transfronterizas en turismo? ¿Existe sensibilización en los actores -stakeholders por el desarrollo del turismo transfronterizo a través de redes, así como la factibilidad de un destino común?

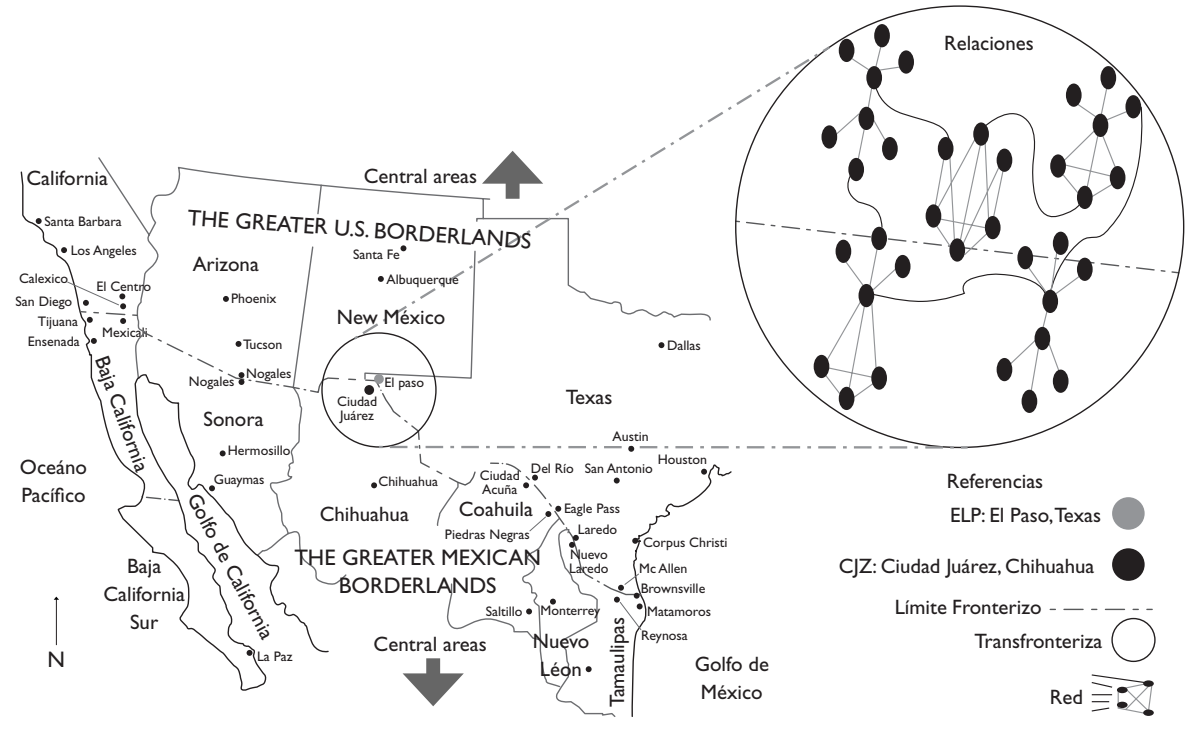

Fuente: Elaboración propia con base en Martínez, [1994: 41].

Figura I. Mapa holístico de la red transfronteriza en la región Juárez-El Paso 
Método de la red transfronteriza

Para la investigación empírica se instrumentó la técnica de "bola de nieve", que identifica a los participantes líderes de opinión de la comunidad (Scout, 2002); al mismo tiempo, distingue a los actores no tan visibles en la sociedad, pero que son reconocidos por su nivel de influencia. En su conjunto constituyen las redes de CJ y ELP graficadas mediante el software UCINET.

Las características del análisis de los datos están diseñadas para determinar el grado de participación de los diversos actores y su diferenciación entre ellos y de la población en general en términos de sus rasgos sociales. Para lograrlo, se llevaron a cabo encuestas semiestructuradas y entrevistas personalizadas, videograbadas, a 36 actores en Cృ y a 32 en ELP, enfocadas a las condiciones del turismo transfronterizo, para explorar lazos de unión de actores y líderes en la actividad a través de redes sociales,y del análisis mediante el software UCINET.

\section{Condiciones del turismo transfronterizo}

La frontera norte de México genera $23.7 \%$ del producto interno bruto nacional, lo que significa $17 \%$ de la población total del país (alrededor de 16.6 millones de personas) y un comercio intenso entre México y Estados Unidos. Por su ubicación geoestratégica, CJ y ELP se han convertido en una de las principales puertas para el intercambio comercial entre estos dos países y Canadá. Por la misma razón, el motivo de viaje de muchos visitantes a esta región es principalmente para negocios (México Now, 2005: 76-77), lo cual equivale a más de $60 \%$ de la ocupación hotelera registrada en los últimos años en la ciudad mexicana (Secretaría de Desarrollo Comercial y Turístico del Gobierno del Estado, 2003); mientras que, en la comunidad paseña, hay una ocupación de $70.5 \%$,y contribuye con $2.4 \%$ del total de ingresos por concepto de turismo en el estado de Texas.

Por otra parte, la apreciación violenta que se tiene de cj no sólo afecta la imagen de la población, sino el entorno social y, por tanto, no permite considerarla como un destino turístico seguro. Al otro lado de la frontera la situación es diferente: se estima que ELP es una de las diez ciudades más seguras de Estados Unidos.

La red cJ refiere un entorno reticular de múltiples relaciones entre stakeholders y actores vinculados en turismo en la comunidad de c (véase figura 2). Las posibles alianzas estratégicas se observan en la configuración de la red, las cuales son fundamentadas en conexiones de vecindad y lazos de unión. Así se 
encuentran representadas por el número de menciones la presidencia municipal, las instituciones de educación superior local (IES), la iniciativa privada, el gobierno del estado, los organismos no gubernamentales, la religión católica, el consulado estadounidense y los organismos empresariales locales.Al mismo tiempo se ofrece un panorama sugestivo de densidad media en el centro de la red.

Se localizaron dos bloques con los vértices más importantes en forma decreciente, representados por los actores con indegree de más mención en c y que los establece como personas líderes. La red de c c tiende más a la centralidad de las relaciones; la permanencia de sus miembros es estable $y$, por tanto, sus lazos son consistentes.

Esta consistencia representa cohesión entre los stakeholders, como se observa en la figura 2. Sin embargo, aunque mayoritariamente integrada en dos bloques, no significa la efectividad de sus relaciones con el entorno turístico. La conformación de los bloques muestra los siguientes atributos:

BLOQUE I: HML (POLÍTICO), JMQS (EDUCACIÓN SUPERIOR), MFI (ORGANISMO NO GUBERNAMENTAL), FV (EMPRESARIO), JB

\begin{tabular}{|c|c|c|}
\hline \multicolumn{3}{|c|}{ (EMPRESARIO-POLÍTICO) } \\
\hline 36 & HML & 0,43961319 \\
\hline 44 & JMQS & 0,39018034 \\
\hline 58 & MFI & $0,337 \mid 3867$ \\
\hline 49 & FV & 0,230686 I I \\
\hline 38 & JB & 0,233551528 \\
\hline 54 & JRB & 0,23067802 \\
\hline $4 I$ & PC & 0,2050195 \\
\hline
\end{tabular}

Bloque 2: RA (IGLESIA), JC (POLÍticoGOBIERNO LOCAL DE ELP), SM (ONG), VF

\begin{tabular}{ccc}
\multicolumn{3}{c}{ (EMPRESARIO) } \\
\hline 35 & RA & DM \\
\hline 35 & 0,19206819
\end{tabular}

55

$J C$

0,18509215

59 SM

0,17576456

60

VF

0,16287986

89 DM

$0,14560 \mid 23$

62

$\mathrm{EF}$

0,13449099

81

DN

0,10104510

101

GV

0,10054394

Fuente: Elaboración propia con base en UCINET.

Figura 2. BLOQUeS RED CJ 
En cuanto a las proporciones de representación en cj, la mención de los políticos locales de esta ciudad es de 1 I \%, mientras los políticos en el nivel estatal y federal alcanzaron $6 \%$, respectivamente. Las IES de CJ y las instituciones de educación superior de ELP obtienen un nivel de $6 \%$, en tanto los empresarios locales tienen una mayor representación de $31 \%$ y los empresarios industriales $6 \%$. Las organizaciones no gubernamentales cuentan con un nivel de $12 \%$, las instituciones religiosas aparecen con $6 \%$, y el gobierno de ELP, con $6 \%$.

La capacidad de influir y el poder de los stakeholders en cj se reflejan en el estatus político-gobierno, que representa $28 \%$, las ONG $12 \%$ y los empresarios $36 \%$; mientras que las IES de CJ y las de ELP, la religión y el gobierno de ELP, muestran $6 \%$. Por su parte, las relaciones transfronterizas CJ-ELP del segundo bloque interactúan con $\mathrm{JCI}$, representante del área de gobierno de ELP, y DNI, actor de las instituciones de educación superior de la misma ciudad.

Respecto a la red ELP, Texas, contiene múltiples relaciones de los stakeholders pero, a diferencia de la red de cJ, ésta ofrece ligas primordialmente con empresarios de la ciudad mexicana. De hecho la red no es tan central porque las menciones van hacia la periferia, de manera que existe sobre todo una escasa difusión entre los interesados e incertidumbre de los esfuerzos. Sin embargo, se nombraron a la alcaldía de ELP, la administración de esa ciudad, los senadores por el estado de Texas, constructores, empresarios de C], asociaciones empresariales locales, emprendedores de turismo local, organizaciones no gubernamentales, el obispado católico y el gobierno del estado de Nuevo México. Las designaciones anteriores constituyen un espectro de mayor alcance y más oportunidades para la actividad del turismo.

Se localizaron tres bloques en ELP con los vértices más importantes en forma decreciente, representados por los actores con indegree de más mención y que los establece como personas líderes de participación. La red, en este caso, muestra una tendencia a la dispersión de los miembros, posiblemente motivada por las inserciones de nuevos ejecutivos de alto nivel. Esta situación conduce a un bajo nivel de compromiso en las interacciones. En la figura 3 se distinguen tres bloques, matizados por la posibilidad de una mayor movilidad, característica común en la cultura estadounidense, y la existencia de otros intereses, los cuales condicionan las relaciones no tan centrales entre los stakeholders. La conformación de los bloques es la siguiente: 
BLOQUE I:JCI (POLÍTICO-GOBIERNO), ES2 (POLÍTICO-SENADOR), WHI (EMPRESARIOCONSTRUCTOR) Y DNI (EDUCACIÓN SUPERIOR)

\begin{tabular}{l|l|l|}
\hline 38 & $\mathrm{JCl}$ & 0,478329549 \\
\hline 55 & $\mathrm{ES} 2$ & 0,374106897 \\
\hline 57 & $\mathrm{WHI}$ & 0,315091352 \\
\hline 29 & $\mathrm{DNI}$ & 0,308634444 \\
\hline
\end{tabular}

BLOQUE 2: BSI (EMPRESARIO-CONSTRUCTOR), JW2 (POLÍTICO-GOBIERNO LOCAL), SRI (POLITICO-ESTATAL) Y PFI (EMPRESARIO COMERCIAL)

\begin{tabular}{l|l|l|}
\hline 43 & BSI & 0,283157522 \\
\hline 40 & JW2 & $0,27538679 I$ \\
\hline 56 & SRI & $0,21027965 I$ \\
\hline 92 & PFI & 0,202165658 \\
\hline
\end{tabular}

BLOQUe 3: GLI (MILITAR NACIONAL), BCI (EMPRESARIO-ORGANISMO EMPRESARIAL), CRD (EMPRESARIO-TURISMO), MDI (EMPRESARIOTURISMO) Y JBI (EMPRESARIO INDUSTRIA-

\begin{tabular}{lll}
\multicolumn{3}{c}{ POLÍTICO CJ) } \\
\hline $5 I$ & GLI & $0,12644 \mid 186$ \\
\hline 52 & BCl & 0,118960048 \\
54 & CRD & 0,118960048 \\
\hline 62 & MDI & 0,116454525 \\
\hline 32 & JBI & $0,1145 \mid 1643$ \\
\hline
\end{tabular}

Fuente: Elaboración propia con base en UCINET.

Figura 3. Bloques Red elp
En proporciones de representación en ELP existe la mención de los políticos locales de $\mathrm{CJ}(6 \%)$, mientras que políticos de la localidad repre149 sentan $15 \%$ al igual que en el nivel estatal, el gobierno local alcanza $7 \%$. Las IES obtienen $8 \%$, los empresarios locales $7 \%$, seguidos de los empresarios industriales con $6 \%$. Las empresas de construcción consiguen mejores niveles con $15 \%$, y los empresarios del ámbito turístico, II \%. Asimismo, la presencia militar alcanza $6 \%$, seguida por los organismos empresariales con $6 \%$.

La capacidad de influir y el poder de los stakeholders en ELP se observa en la representación del estatus político-gobierno que constituye $42 \%$, las IES $8 \%$, los empresarios en general $38 \%$, los militares $6 \%$ y las cámaras empresarial otro $6 \%$. En tanto, las relaciones transfronterizas de ELP-CJ se ubican en el bloque 3 e interactúan con JBI de representante empresarial y político de CJ.

Los significativos cambios acaecidos en los últimos años han modificado el panorama del mercado turístico mundial y lo han enfrentado a nuevos retos y oportunidades (Lillo Bañuls, Ramón Rodríguez y Sevilla Jiménez 2007). Estos cambios evidencian la necesidad de mejorar la gestión de los recursos disponibles, sobre todo en zonas fronterizas 
como la de México-Estados Unidos, porque se encuentran caracterizadas por conflictos sociopolíticos y socioeconómicos que afectan el ámbito turístico. Tal es el caso de la frontera CJ-ELP. Esta compleja situación se ve reflejada en la conformación de redes estratégicas entre ambas comunidades; así es como:

- La retícula de c muestra una compacta mención; sólo cuenta con dos bloques (véase figura 4), lo que indica cohesión y lazos de unión. A la par, exhibe una marcada capacidad de influencia y poder por stakeholders en las esferas políticas y de gobierno en los tres niveles ( $28 \%$ ); el empresariado en su conjunto, mayoritariamente del comercio (36\%); mientras

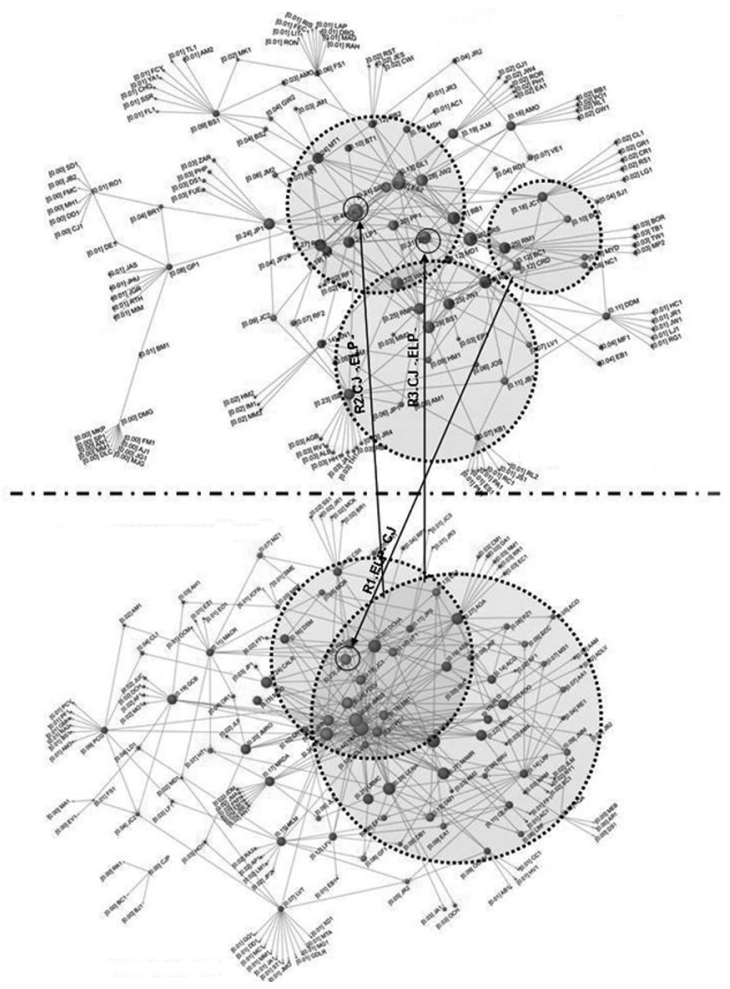

Fuente: Elaboración propia con base en UCINET.

FIGURA 4. INTERACCIONES ENTRE ACTORES CJ-ELP 
que los stakeholders de la educación superior (IES) y la religión muestran $6 \%$, y los organismos no gubernamentales, $12 \%$. Es sugestivo el resultado de la participación de las ONG en el panorama general, que puede ser interpretado por la búsqueda de un equilibrio en relación con el gobierno y la política, ámbitos muy desacreditados. Sin embargo, visiblemente no existe participación del empresariado en turismo, lo que apoya la idea de la insuficiente relación con los ámbitos de poder social y económico. Otro detalle significativo es la presencia de la religión como actor relevante en la sociedad, aunque con baja representación. Por otra parte, la educación superior (IES) y la alcaldía de ELP se encuentran en las menciones de la red de $\mathrm{C}$.

- La retícula de elp muestra una mayor dispersión en la mención; exhibe tres bloques muy diversificados en su configuración y, a la vez, muy equilibrados. En lo relativo a la capacidad de influir y de poder, se observa una prevalencia de la esfera política y de gobierno ( $42 \%)$, seguida del empresariado en general (32\%) y una equilibrada presencia de la educación superior, organismos empresariales y militares. Esta última constata la distribución de la comunidad con las actividades bélicas, empero, dentro del empresariado destaca la participación de los empresarios en turismo con I I \% del total, reforzando la consideración de la sensibilidad por la actividad turística.

\section{Conclusiones}

La actividad turística, sin duda, es un sistema complejo que requiere participación entre los stakeholders a través de sus líderes, la cual debe extenderse entre las comunidades en el área de influencia de CJ-ELP. Sin embargo, las redes identificadas muestran una brecha entre los stakeholders en turismo de ambas comunidades fronterizas, lo que significa un fundamento para la constitución de una red transfronteriza, partiendo de lazos débiles, a consecuencia de la dureza del poder hegemónico estadounidense; puesto que el manejo del poder es un elemento invariable en cualquier contexto estratégico.

De aquí la necesidad imperante de comprender los "rasgos de una red transfronteriza" para lograr transacciones dentro de un contexto de reciprocidad y relaciones de interdependencia socioeconómica. Desde la concepción del territorio ocupado por una población fronteriza, unida por lazos históricos 
y culturales, con potencial para el desarrollo turístico, Delgadillo y Torres argumentan que

...esta ocupación depende y se relaciona con los procesos económicos y sociales, mismos que de no considerarse, así sea mediante el acuerdo más elemental, ocurriría un caos territorial que terminaría por destruir significativamente el entorno, antes de explorar siquiera su potencial [2006: 2].

Ello ha supuesto la insuficiencia del actual modelo en ambas comunidades; la necesidad de explorar nuevos estrategias para la región y sondear las probabilidades del planteamiento de Jiménez (1998) relativo a los tipos de ciudades con contexto turístico, entre aquellas urbes que son impactadas de forma parcial y aquellas que dependen de la actividad para su sostenimiento. Pero no se debe negar el crecimiento de ambas comunidades; si bien ha sido exponencial en c], en términos de servicios y población, cabe acotar que éste ha ocurrido por las necesidades del modelo económico, fundamentado en la maquiladora; mientras que el crecimiento en ELP, se debe en primer instancia a la base militar de Fort Bliss, y a la interdependencia socioeconómica con Juárez.

Finalmente, persiste el dilema de cómo afrontar esta convivencia en ciudades fronterizas, porque el tejido de la actual red transfronteriza en turismo se encuentra en el escenario "noción reticular local e influencia fronteriza" de manera incipiente. Por tanto, queda para futuras investigaciones ahondar más en la interdependencia compleja, en la esfera del turismo transfronterizo en red, porque ofrece profundizar en los desafíos relativos a una cooperación y colaboración en la frontera mediante el proceso de la sociabilidad.

\section{FUENTES CONSULTADAS}

Aghón, G.l. et al. (200I). Desarrollo económico local y descentralización en América Latina: un análisis comparativo. Santiago de Chile: Comisión Económica para América Latina y el Caribe.

Bodin, J. (2006). Los seis libros de la República. Madrid:Tecnos.

Bolaños, L. (200I). La identidad perdida y otros mitos. México:Vila.

Bourdieu, Pierre (2000). Intelectuales, política y poder. Buenos Aires: Eudeba.

Burt, Ronald S. ( 1982). Toward a Structural Theory of Action. Nueva York:Academic Press. 
Coleman, James S. (1994). Foundations of Social Theory. Cambridge: Harvard University Press.

Dabas, E. y D. Najmanovich (comps.) (1995). Redes. El lenguaje de los vínculos. Buenos Aires: Paidós.

De Nooy, W., A. Mrvar y V. Batagelj (2005). Exploratory Social Network. Analysis with Pajek. Ljubljana: University of Ljubljana/ESNA.

De Rojas, Carmen y Carmen Camarero (2008). 'Visitors' Experience, Mood and Satisfaction in a Heritage Context: Evidence from an Interpretation Center”. Tourism Management, 29 (3), 525-537. Disponible en: http:// www.sciencedirect.com [2008, junio].

Delgadillo, J. y F.Torres (2006). Dimensiones multicausales del ordenamiento territorial. Enfoques y aplicaciones. México: Instituto de Investigaciones Económicas de la Universidad Nacional Autónoma de México/Sistema Nacional de Investigadores, mimeo.

Diccionario Ideológico de la Lengua Española (1998). Barcelona:Vox.

Elías, Norbert (200I). The Society of Individuals. Nueva York: Continuum.

European Communities (2006). Innovation in Tourism. How to Create aTourism Learning Area.The Handbook. Bélgica: European Commission, Tourism Unit. Fernández, S. (1995). “La red como alternativa de desarrollo comunitario”, en E. Dabas y D. Najmanovich (comps.). Redes. El lenguaje de los vínculos. Buenos Aires: Paidós, 395-40I.

Freeman, L.C. (1968). Patterns of Local Community Leadership. Indianápolis:The Bobbs-Merrill.

Geddes, M. (2005). Making Public Private Partnerships Work. Hants: Gower. Giménez, Gilberto (2007)."La frontera norte como representación y referente cultural en México”. Cultura y representaciones sociales, año 2, núm. 3, septiembre. Disponible en: http://www.culturayrs.org.mx/revista/ num3/Gimenez.pdf [2009, marzo].

Gledhill,John (1999)."El reto de la globalización: reconstrucción de identidades, formas de vida transnacionales y las ciencias sociales", en Gail Mummert (ed.). Fronteras fragmentadas. Zamora: El Colegio de Michoacán, Centro de Investigación y Desarrollo del Estado de Michoacán, 23-54.

Hanneman, R.A. (2002). Introduction to Social Network Methods. Riverside: Department of Sociology, University of California. 
loannides, D., P.A. Nielsen y P. Billing (2006). "Transboundary Collaboration in Tourism: The Case of the Bothnian Arc”. Tourism Geographies, 8 (2), I22-142.

Jiménez,A. (1998). Desarrollo turístico y sustentabilidad: el caso de México. México: Porrúa. Disponible en: http://dzibanche.biblos.uqroo.mx/Cursos_ linea/Efrain_Villanueva/Otono200I/EICasoMexico.htm [2007, julio]. Keohane, R. y J. Nye (1988). Poder e interdependencia. La política mundial en transición. Buenos Aires: Grupo Editorial Latinoamericano.

Kickert,W.J.M., E-H. Klijn y F.M. Koppenjan ( 1997). Managing Complex Networks. Strategies for the Public Sector. Londres: SAGE.

L'Etang, J., J. Falkheimer y J. Lugo (2007). "Public Relations and Tourism: Critical Reflections and a Research Agenda”. Public Relations Review, 33 (I), 68-76. Disponible en: http://www.sciencedirect.com [2007, julio]. Lagiewski, R. M.y Revelas, D.A. (2002). Challenges in Cross-BorderTourism Regions. Rochester y Dubrovnik: Rochester Institute of Technology/American College of Management \& Technology. Disponible en http://hdl.handle. net//850//603 [2007, agosto].

Lillo Bañuls, Adelaida, Ana Belén Ramón Rodríguez y Martín Sevilla Jiménez (2007). "El capital humano como factor estratégico para la competitividad del sector turístico". Cuadernos de Turismo, 19, enero-junio, 47-69. Murcia: Universidad de Murcia. Disponible en: http://redalyc. uaemex.mx [2007, julio].

Martínez, Oscar J. ( 1982). Ciudad Juárez: el auge de una ciudad fronteriza a partir de 1848. México: Fondo de Cultura Económica.

(1994). Border People: Life and Society in the U.S.-Mexico Borderlands. Tucson y Londres:The University of Arizona Press.

Méndez, Eloy e Isabel Rodríguez (2004)."Comunidades cercadas en la frontera México-EEUU”. Scripta Nova. Revista Electrónica de Geografia y Ciencias Sociales, VIII (I7I), I 5 de agosto de 2004. Barcelona: Universidad de Barcelona. Disponible en: http://www.ub.es/geocrit/sn/sn- I 7I.htm México Now (2005). México Now, III (I6), mayo-junio de 2005, 76-77. Moliner, M. (1998). Diccionario de uso del español. Madrid: Gredos.

Motta, R. (I995). "Las redes sociales informales y la búsqueda de la ecuación interactiva entre la toma de decisiones locales y la responsabilidad de la gobernabilidad global", en E. Dabas y D. Najmanovich (comps.). Redes. El lenguaje de los vínculos. Buenos Aires: Paidós, 373-38I. 
Nye, J.S. (2002). La parodia del poder norteamericano. Madrid:Taurus.

Palmer, C. (1999). "Tourism and the Symbols of Identity". Tourism Management, 20 (3), 3 I 3-32 I. Disponible en: http://www.sciencedirect.com [2007, agosto].

Pechlaner, H., Abfalder, D. y Raich, F. (2002). "Cross-Border Destination Managment Systems in the Alpine-Region. The Role of Knowledge Networks on the Example of AlpNet", en Bouncken, R. B.y Sungsoo, P. (Eds.). Knowledge Management in Hospitality andTourism. Nueva York: The Hawort Press.

Prokkola, Eeva-Kaisa (2007). “Cross-border Regionalization and Tourism Development at the Swedish-Finnish Border:'Destination Arctic Circle”". Scandinavian Journal of Hospitality and Tourism, 7 (2), I 20-I 38.

Secretaría de Desarrollo Comercial y Turístico del Gobierno del Estado (2003). Dirección de Turismo / Departamento de Planeación, Chihuahua, 2003.

Scott, J. (2002) Social Network Analysis. SAGE, London.

Timothy, D.J. (1995). "Political Boundaries and Tourism: Borders as Tourist Attractions". Tourism Management, 16 (7), 525-532.

Timothy, D.J.y R.W. Butler (1995).“Cross-Border Shopping.A North American Perspective". Annals of Tourism Research, 22 (I), 16-34.

Trevor, H. y B. Sofield (2006). "Border Tourism and Border Communities: An Overview”. Tourism Geographies, 8 (2), I02-I2I.

Vázquez Barquero,A. ( 1999). Desarrollo, redes e innovación. Lecciones sobre desarrollo endógeno. Barcelona: Pirámide (Economía y Empresa).

Watts, Duncan J. (1999). SmallWorlds. Nueva Jersey: Princeton University Press. Welch, E.yWong,W. (200I)."Effects of Global Pressures on Public Bureaucracy: Modeling a New Theoretical Framework". Administration y Society, 33 (4), pp. 37I-402.

Werthner, H.y Klein, S. ( 1999). Information Technology and Tourism - A Challenging Relationship.Austria: Springer Wein New York. 